\title{
KU LEUVEN
}

\begin{tabular}{|c|c|}
\hline Citation & $\begin{array}{l}\text { Miner PD, Canobbio MC, Pearson DD, Schlater M, Balon Y, Junge KJ, Bhatt } \\
\text { A, Barber D, Nickolaus MJ, Kovacs AH, Moons P, Shaw K, Fernandes SM } \\
\text { Contraceptive practices of women with complex congenital heart disease } \\
\text { American Journal of Cardiology }\end{array}$ \\
\hline Archived version & $\begin{array}{l}\text { Author manuscript: the content is identical to the content of the published } \\
\text { paper, but without the final typesetting by the publisher }\end{array}$ \\
\hline Published version & Klik hier als u tekst wilt invoeren. \\
\hline Journal homepage & \\
\hline Author contact & $\begin{array}{l}\text { your email Philip.Moons@kuleuven.be } \\
\text { your phone number + } 32(0) 16373315\end{array}$ \\
\hline IR & \\
\hline
\end{tabular}

(article begins on next page) 


\section{Contraceptive Practices of Women with Complex Congenital Heart Disease}

Pamela D. Miner, RN, MN, NP a; Mary M. Canobbio, RN, MN, CNS ${ }^{\text {; }}$; Dorothy D. Pearson, PA-C b; Mary Schlater, RN, ARNP c; Yvonne Balon, RN, MN d; Kathryn J. Junge, RN, BSNe; Ami Bhatt, MDf; Deena Barber BSN, RNg; Michelle J. Nickolaus, MSN, CRNPh; Adrienne H Kovacs, PhD, CPsychi; Philip Moons, RN, PhDi; Kate Shaw, MD, MS'; Susan M. Fernandes, LPD, PA-C

\footnotetext{
${ }^{a}$ Ahmanson/UCLA Adult Congenital Heart Disease Center, UCLA Medical Center, Los Angeles CA

${ }^{b}$ Boston Adult Congenital Heart Program, Boston Children's Hospital, Boston MA

${ }^{c}$ Adult Congenital Heart Disease Clinic, University of Washington Regional Heart Center, Seattle WA d Adult Congenital Heart Clinic, Peter Lougheed Hospital, Calgary AB

${ }^{\mathrm{e}}$ Center for Adults with Congenital Heart Disease, Washington University School of Medicine, Barnes Jewish Hospital, St. Louis MO

f Congenital Heart Disease Program, Massachusetts General Hospital, Harvard Medical School, Boston MA

g Adult Congenital Heart Service Akron Children's Hospital, Akron OH

h Penn State Hershey Program for Adult Congenital Heart Disease, Hershey PA

i Toronto Congenital Cardiac Centre for Adults, Toronto General Hospital, Toronto, Ontario Canada

j KU Leuven Department of Public Health and Primary Care, KU, Leuven, University of Leuven, Belgium

${ }^{k}$ Stanford University, Department of Obstetrics \& Gynecology, Palo Alto, CA

I Stanford University, Departments of Pediatrics and Medicine, Divisions of Pediatric Cardiology and Cardiovascular Medicine, Palo Alto, CA
}

Address for correspondence: $\quad$ Pamela D. Miner, RN, MN, NP

T: 310-825-2019/F: 310-825-6346

pminer@mednet.ucla.edu

Ahmanson/UCLA Adult Congenital Heart Disease Center

UCLA Cardiovascular Center

100 UCLA Medical Plaza, Suite 630 East

Los Angeles, CA 90095

There are no relationships with industry

Brief Title: Contraception in Women with Congenital Heart Disease 


\begin{abstract}
Understanding the contraceptive practices of females with complex congenital heart disease (CHD) and providing them individualized contraception counseling may prevent adverse events and unplanned high risk pregnancies. Given this, we sought to examine the contraceptive practices in women with CHD, describe adverse events associated with contraceptive use, and describe the provision of contraception counseling. Women $>18$ years of age were recruited between 2011 and 2014 from 9 adult CHD (ACHD) centers throughout North America. Subjects completed a 48-item questionnaire regarding contraceptive use and perceptions of contraception counseling, and a medical record review was performed. Of 505 subjects, median age was 33 (IQR, 26 to 44 ) and $81 \%$ had CHD of moderate or great complexity. The majority $(86 \%, 435 / 505)$ of the cohort had utilized contraception. The types included: barrier methods (87\%), oral contraception (OC) $84 \%$, intra-uterine device (18\%), Depo-Provera (15\%), vaginal ring (7\%), patch (6\%), hormonal implant (2\%), Plan B (19\%), and sterilization (16\%). Overall OC use was not significantly different by CHD complexity. Women with CHD of great complexity were more likely to report a thrombotic event (TE) while taking OC than those with less complex $\mathrm{CHD}$ ( $9 \%$ vs.1\%, $\mathrm{p}=0.003$ ). Contraception counseling by the ACHD team was noted by $43 \%$ of subjects. Unplanned pregnancy was reported by $25 \%$ with no statistical difference by CHD complexity. In conclusion, contraceptive practices of women with complex CHD are highly variable, and the prevalence of blood clots while taking $O C$ is not insignificant while provision of contraception counseling by ACHD providers appears lacking.
\end{abstract}

Key Words: congenital heart disease, contraception, pregnancy, Fontan, thrombosis 


\section{INTRODUCTION}

Today, $>90 \%$ of children born with congenital heart disease (CHD) are expected to live well into adulthood [1, 2], and epidemiological studies estimate that as many as $9-15 \%$ of adult CHD survivors have the most complex forms of $\mathrm{CHD}[3]$. As a result, the number of women with complex CHD reaching childbearing age has also increased. Because of the documented risk of pregnancy in women with complex CHD [4-7], pre-conception counseling that includes discussion of safe and effective contraceptive options is considered to be an integral component of health maintenance $[8,9]$. However, data regarding contraceptive practices of females with more complex forms of CHD, including side effects and adverse events related to contraception, as well as the education and counseling given by health care providers, is limited. Given this, we sought to describe the contraceptive practices of women followed by adult $\mathrm{CHD}(\mathrm{ACHD})$ centers throughout North America.

\section{METHODS}

A cross-sectional survey of women $\geq 18$ years of age with CHD was conducted between 2011 and 2014 at 9 ACHD centers throughout North America. Subjects were recruited either in person during an outpatient clinic visit, or invited to participate by email or telephone. Patients were asked to complete a structured self-administered questionnaire regarding contraceptive use, side effects of contraceptives, adverse medical events, reasons for stopping contraceptive method, unplanned pregnancies, age at menarche and menstrual patterns, and who provided contraception counseling.

Once informed consent was obtained, patients were given the option of completing a questionnaire either on paper or online. Medical records were reviewed to obtain primary 
cardiac diagnosis, surgical history, pharmacologic treatment, and comorbidities, including thrombotic history and oxygen saturation. Additionally, medical records were reviewed for evidence of contraception and/or pregnancy counseling.

The institutional review board of the 9 participating centers approved the study protocol. Complexity of CHD lesions/surgeries were categorized as simple complexity, moderate complexity and great complexity; according to 2008 ACC/AHA Guidelines for the Management of Adults with Congenital Heart Disease [2].

We provide descriptive analyses summarized as medians and interquartile range (IQR) for continuous variables and proportions for categorical variables. Center, clinical, and demographic differences were analyzed using Pearson's chi-square or Fisher's Exact test for categorical variables and Mann-Whitney $\mathrm{U}$ or Kruskal Wallis tests for continuous variables. A sub-analysis was performed in a group of subjects deemed by prior literature to be at increased risk for thrombosis and thromboembolism with the use of estrogen-based contraception $[8,9]$. This sub-analysis assessed their use of estrogen-based contraception and the prevalence of thrombus, both reported and/or based on medical records. This high-risk group included subjects with mechanical valves, subjects with current cyanosis, and those who had undergone the Fontan operation. A 2-sided $p$-value of $<0.05$ was considered to indicate statistical significance. All analyses were performed using SPSS software version 23 (Chicago, IL).

\section{RESULTS}

A total of 505 of $510(98 \%)$ women agreed to study participation. Characteristics of the study cohort are summarized in Table 1 . The median age at menarche was 13 (IQR, 12 to 14). Of the women in this cohort still menstruating (80\%), the frequency of menses was between 25 
and 28 days for most (47\%). Only $16 \%$ reported heavy bleeding during menses. There was no significant difference in the frequency or length of menses or complaints of heavy bleeding by CHD complexity. In women who reported no longer menstruating (median age 50 [IQR, 41.8 to 50] years), the most common reason was being post-menopausal (43\%) or having had a hysterectomy (27\%). Gynecological issues including an abnormal pap smear, ovarian cyst, and sexually transmitted infection were reported by $23 \%, 20 \%$ and $16 \%$ respectively. There were 11 (2\%) women who reported having had a cancer (breast 5, cervical 4, uterine 1 and ovarian 1). Only $6 \%$ of the subjects reported a history of infertility and this did not vary by disease complexity.

Contraception use by disease complexity is reported in Table 2. After barrier method, oral contraception (OC) was the most common form of birth control. The mean length of OC use was 4 years (IQR, 1 to 9) and the most common symptoms associated with use was headaches (35\%), bloating (33\%), cramping (30\%), and weight gain (31\%). Only 5\% reported lower-extremity edema. Rates of OC use were similar for each CHD complexity group, however women with CHD of great complexity were less likely to use a combination estrogen and progestin type $O C$ and more likely to use progestin-only type OC than those with less complex CHD.

Thrombosis/thromboembolic (T/TE) events while using OC were reported by $4 \%$ of cohort. The CHD diagnoses for these women included bicuspid aortic valve $(n=1)$, repaired complete atrioventricular canal defect $(n=2)$, Eisenmenger syndrome $(n=2)$, D-transposition of the great arteries with Mustard or Senning repair $(n=4)$, and Fontan physiology $(n=5$, including 3 with lateral tunnel, 1 with extra-cardiac conduit, 1 with right atrium to pulmonary artery). 
Women with CHD of great complexity were more likely to have reported having T/TE events while taking $\mathrm{OC}$ than those with less complex $\mathrm{CHD}$ ( $9 \%$ versus $1 \%, \mathrm{p}=0.003$ ).

Of the 31 women with Fontan physiology, $58 \%$ had used or were currently using OC ( $50 \%$ progestin, $61 \%$ combination OC, and $17 \%$ third-generation OC). Of these women who had used OC, $28 \%(5 / 18)$ reported having a T/TE event while taking OC. An intracardiac thrombus was noted in 3 women, with 1 of these patients also having a pulmonary embolus. In addition, there was 1 other patient who had a pulmonary emboli and 1 who had a transient ischemic attack (TIA). The type of contraception being utilized at the time of the event was a combination (estrogen/progestin) OC in 4 and a third-generation combination OC in 1 . The prevalence of $\mathrm{T} / \mathrm{TE}$ events in those patients who had never used OC was $17 \%$, compared to $39 \%$ in those who had used OC. Only 1 patient was known to be on anti-coagulation at the time of event, but the INR result at the time of event was not available.

There were 38 women with D-transposition of the great arteries with atrial switch repair (Mustard or Senning), 82\% (31/38) of whom reported having previously used OC. Of these women, 4/31 (13\%) reported having a T/TE event while taking OC. A lower-extremity clot was reported in 2 patients and 2 had a TIA. The type of OC used at the time of event was progestinonly in 1 and combination in $1 ; 2$ women were unable to recall the type of $O C$ they were on at the time of the event. The women who reported never taking an OC had no reported history of a T/TE by either report or chart review.

A total of 27 (5\%) women had 1 or more mechanical valves (AVR 16, MVR 6, TVR 3, PVR 3). Of this group $74 \%(20 / 27)$ reported taking OC ( $53 \%$ progestin-only, $68 \%$ combination and $1 \%$ third-generation), and none of these women reported a blood clot during use. 
Alternative contraceptive methods including IUDs, Depo Provera injection, Plan B emergency contraception and sterilization for pregnancy prevention were each utilized by less than a quarter of the sample (see table 2). The median age of tubal sterilization was 28 (IQR 24 to 31.2 ) years. Women with CHD of great complexity were more likely to undergo sterilization compared to those less complex groups ( $22 \%$ versus $12 \%, p=0.047)$. Women with CHD of great complexity were more likely to have undergone sterilization for cardiac indications than those with less complex CHD (74\% [26/35] versus $42 \%$ [14/33], $p=0.003)$. The male partner of $9 \%$ of the women in this study underwent a vasectomy for pregnancy prevention.

The majority of women in our cohort $(84 \%, 424 / 505)$ reported receiving contraception counseling, but only $43 \%(216 / 505)$ indicated receiving counseling from their ACHD provider (physician, nurse practitioner, or physician assistant). Gynecologists were the source of contraception counseling for $55 \%$ (280/505), and primary care physicians were least likely to provide counseling $(11 \%, 56 / 505)$. There were no differences in disease complexity or marital status of women who reported receiving contraception counseling by their ACHD provider. However, Hispanic women were less likely to report receiving such information compared to non-Hispanic women ( $69 \%$ versus $89 \%, p=0.001$ ), as were older women compared to younger women (39.0 \pm 13.6 versus $34.8 \pm 11.2, p=0.027)$. Patients with CHD of great complexity were more likely to have documentation of contraception counseling than patients with less complex CHD $(56.0 \%$ versus $45 \%, p=0.036)$.

\section{DISCUSSION}

Understanding the current contraceptive practices of women with ACHD and providing 
individualized contraception counseling is necessary to prevent adverse events associated with contraception and prevent unplanned high risk pregnancies. This multi-center study examined the contraceptive practices of more than 500 women, the majority of whom had CHD of moderate or great complexity. The main findings of our study suggest that the ACHD population utilizes the full spectrum of contraception, with OCs being the preferred method after barrier methods. Despite guidelines recommending against estrogen-based contraception in some high-risk ACHD patients [2], this type of therapy was not uncommonly used, and the reported incidence of T/TE events in these patients is higher than expected and requires further investigation. Less than half of our cohort reported receiving contraception counseling from their ACHD provider, despite all subjects being recruited from ACHD specialty centers.

Contraceptive options have evolved over the past few decades, providing women with multiple options for effective pregnancy prevention. Current national and international contraception guidelines have led to recommendations regarding the first-line choice for effective birth control for all adolescent and adult women, including those women considered at higher thrombotic risk, and that choice is the IUD or progestin implants $[12,13]$. The use of IUDs and progestin implants has steadily increased in the U.S. over the past decade, with an estimated $11 \%$ of women currently using one of these methods of long-acting reversible contraception $[13,14]$. The use of IUDs by this cohort was much higher than the national average at $18 \%$, while implantable progestin was only used by $2 \%$. Emergency contraception was utilized more frequently by women in this study compared to the general U.S. population (19\% versus 11\%) [ 14]. Sterilization (tubal occlusion, vasectomy) was used at similar rates to the national average [15]. 
Despite the wide use of contraception by the women in this study, $25 \%$ of subjects reported an unplanned pregnancy. Although this is lower than the national average at $49 \%$ [16], patients with CHD of great complexity were as likely to have an unplanned pregnancy as those with simple CHD defects. Although this study did not specifically ask subjects if unplanned pregnancies were a result of not using contraception while being sexually active, previous studies report that more than a quarter of female patients with CHD admit to not using any type of contraception while being sexually active [9].

The use of OCs as the birth control method of choice for women with CHD is consistent with contraceptive preferences in the U.S [17]. The risk for T/TE events associated with estrogen based $\mathrm{OC}$ is well documented. Given this risk, consensus recommendations have guided providers to avoid estrogen-containing contraceptives in ACHD patients known to be at increased risk of T/TE events [10,11]. This includes women with Fontan physiology, Eisenmenger syndrome/cyanosis, and mechanical heart valves [11]. Despite these recommendations, a significant number of "high-risk" subjects in this cohort were currently using or had used estrogen-containing contraceptives. This finding is consistent with a study by Vigl et al., who reported that $20 \%$ of their study sample was using contraceptive methods contraindicated for their cardiac condition [9].

In this study $4 \%$ of subjects reported and/or were documented to have had a T/TE event while on OC. This is higher than what we would expect to see in the general population [18], but similar to the $3.8 \%$ incidence of T/TE events in CHD women noted by Pijuan-Domenech \& colleagues [19]. In our cohort, the subjects that reported a T/TE event while on OCs included those expected to be at high risk, such as those with Fontan physiology and Eisenmenger 
syndrome. The Fontan group appeared to be at particular risk, with $28 \%$ of the subjects who had used OCs reporting a T/TE event. Given the study design and small number of patients $(n=31)$, we cannot conclude a cause-and-effect relationship between $O C$ use and T/TE events in Fontan patients. However, we know from previous studies that those with Fontan physiology are at significant risk for T/TE events (10-20\%) even without a provocative agent [20]. As stated, we found that T/TE events were higher in women with Fontans who had used OC compared to those who had not (39\% versus $17 \%)$. This finding certainly raises additional questions for this population, and warrants further study. Of interest, we noted T/TE events in 4/31 patients with atrial switch (Mustard/Senning) repair for D-transposition of the great arteries while using OC. This group has not been considered particularly high risk for T/TE in the past, although paradoxical emboli related to intracardiac baffle leaks are well documented [21]. Given our findings, baseline T/TE risk should be considered prior to initiating estrogenbased contraception in these patients.

Contraception counseling should be an integral part of any adolescent or ACHD program, regardless of whether or not a patient is known to be sexually active. The literature recognizes deficiencies in counseling CHD women with pregnancy and/or contraceptive risks. Vigl et al. found that only a little more than half of ACHD patients in Germany had received information regarding pregnancy or contraceptive options and their associated risks [9]. Similarly, Hinze et al. reported that more than half of their sample of women with CHD had not received contraception counseling, and those at high risk were using methods that were contraindicated for their cardiac condition [8]. Our study found that $84 \%$ of the patients reported receiving some contraception counseling; however, only $43 \%$ reported that this advice 
came from their ACHD providers. Given this information, ACHD specialists need to ensure that all CHD women receive individualized information about their contraceptive options and are aware of their risk in regards to both contraceptive use and unplanned pregnancy. In addition, the ACHD community needs to ensure that primary care providers and gynecologists are educated about the need to work with their ACHD team to determine the best contraceptive options for their CHD patients

This study has several limitations, most notably selection bias, as all patients were being followed in an ACHD specialty center at the time of recruitment. In addition, our population was predominately white and highly educated. Given this, results may not be generalizable to the greater population of ACHD patients. The self-reporting nature of the study likely results in some recall bias; however, all T/TE events were verified by medical record review. Social desirability bias may have also impacted some of the results, especially around topics such unplanned pregnancy and sexually transmitted diseases. 
Table 1. Baseline Characteristícs by Congenital Heart Disease Complexity

\begin{tabular}{lcccc}
\hline \multicolumn{1}{c}{ Characteristic } & $\begin{array}{c}\text { All } \\
(\mathrm{N}=\mathbf{5 0 5})\end{array}$ & $\begin{array}{c}\text { Simple } \\
\text { Complexity } \\
(\mathrm{N}=95)\end{array}$ & $\begin{array}{c}\text { Moderate } \\
\text { Complexity } \\
(\mathrm{N}=\mathbf{2 2 7})\end{array}$ & $\begin{array}{c}\text { Great } \\
\text { Complexity } \\
(\mathbf{N}=\mathbf{1 8 3})\end{array}$ \\
\hline Age (median, IQR) (years): & $33(26$ to 44$)$ & $31(25$ to 43$)$ & $35(26$ to 45$)$ & $32(27$ to 40.5$)$ \\
Married & $220(44 \%)$ & $41(43 \%)$ & $105(46 \%)$ & $74(40 \%)$ \\
Hispanic & $33(7 \%)$ & $3(3 \%)$ & $16(7 \%)$ & $14(8 \%)$ \\
White & $418(83 \%)$ & $76(80 \%)$ & $192(85 \%)$ & $150(82 \%)$ \\
Education $>$ Associate Degree & $298(59 \%)$ & $54(57 \%)$ & $138(61 \%)$ & $106(58 \%)$ \\
\hline
\end{tabular}


Table 2. Contraception Use by Congenital Heart Disease Complexity

\begin{tabular}{|c|c|c|c|c|c|}
\hline Characteristic & $\begin{array}{c}\text { All } \\
(N=505)\end{array}$ & $\begin{array}{c}\text { Simple } \\
\text { Complexity } \\
(\mathrm{N}=95)\end{array}$ & $\begin{array}{c}\text { Moderate } \\
\text { Complexity } \\
(\mathrm{N}=227)\end{array}$ & $\begin{array}{c}\text { Great } \\
\text { Complexity } \\
(\mathrm{N}=183)\end{array}$ & P-Value \\
\hline Any type of contraception & $435(86 \%)$ & $80(84 \%)$ & $196(86 \%)$ & $159(87 \%)$ & 0.922 \\
\hline Oral Contraception & $365(84 \%)$ & $69(86 \%)$ & $165(84 \%)$ & $131(82 \%)$ & 0.747 \\
\hline Progestin & $108(30 \%)$ & $13(19 \%)$ & $44(27 \%)$ & $51(39 \%)$ & 0.034 \\
\hline Combination & $248(68 \%)$ & $56(81 \%)$ & $122(74 \%)$ & $70(53 \%)$ & $<0.001$ \\
\hline $3^{\text {rd }}$ Generation Combination & $52(14 \%)$ & $11(16 \%)$ & $26(16 \%)$ & $15(12 \%)$ & 0.517 \\
\hline Intrauterine Device & $80(18 \%)$ & $13(16 \%)$ & $34(17 \%)$ & $33(21 \%)$ & 0.708 \\
\hline Vaginal Ring & $29(7 \%)$ & $8(10 \%)$ & $10(5 \%)$ & $11(7 \%)$ & 0.404 \\
\hline Patch & $28(6 \%)$ & $6(8 \%)$ & $11(6 \%)$ & $11(7 \%)$ & 0.929 \\
\hline Depo Provera & $63(15 \%)$ & $9(11 \%)$ & $25(13 \%)$ & $29(18 \%)$ & 0.339 \\
\hline Progestin Implant & $8(2 \%)$ & $1(1 \%)$ & $4(2 \%)$ & $3(2 \%)$ & 0.839 \\
\hline Barrier Method & $377(87 \%)$ & $69(87 \%)$ & $173(88 \%)$ & $135(85 \%)$ & 0.359 \\
\hline Tubal Sterilization & $68(14 \%)$ & $7(9 \%)$ & $26(13 \%)$ & $35(22 \%)$ & 0.047 \\
\hline Emergency Contraception & $81(19 \%)$ & $14(18 \%)$ & $44(22 \%)$ & $23(15 \%)$ & 0.212 \\
\hline
\end{tabular}


[1] Moons P, Bovijn L, Budts W, Belmans A, Gewillig $M$. Temporal trends in survival to adulthood among patients born with congenital heart disease from 1970 to 1992 in Belgium. Circulation 2010; 122: 2264-2271.

[2] Warnes CA, Williams RG, Bashore TM, Child JS, Connolly HM, Dearani JA, del Nido P, Fasules JW, Graham Jr TP, Hijazi ZM, Hunt SA, King ME , Landzberg MJ, Miner PD , Radford MJ, Walsh EP, Webb GD. ACC/AHA 2008 Guidelines for the Management of Adults With Congenital Heart Disease: A Report of the American College of Cardiology/American Heart Association Task Force on Practice Guidelines (Writing Committee to Develop Guidelines for the Management of Adults With Congenital Heart Disease): Developed in Collaboration With the American Society of Echocardiography, Heart Rhythm Society, International Society for Adult Congenital Heart Disease, Society for Cardiovascular Angiography and Interventions, and Society of Thoracic Surgeons. Circulation 2008; 118: e714-e833.

[3] Marelli AJ, Mackie AS, lonescu-Ittu R, Rahme E, Pilote L. Congenital heart disease in the general population: changing prevalence and age distribution, Circulation 2007; 115: 163-172.

[4] Canobbio MM, Mair DD, van der Velde M, Koos BJ. Pregnancy outcomes after the Fontan repair. J Am Coll Cardiol 1996: 28; 763-767.

[5] Canobbio MM, Morris CD , Graham TP, Landzberg MJ. Pregnancy outcomes after atrial repair for transposition of the great arteries. Am J Cardio/ 2006: 98; 668-672. 
[6] Greutmann M, Silversides CK. The ROPAC registry: a multicentre collaboration on pregnancy outcomes in women with heart disease. Eur Heart J 2013: 34; 634-635.

[7] Tobler D, Fernandes SM, Wald RM, Landzberg M, Salehian O, Siu SC, Colman JM, Sermer M , Silversides CK. Pregnancy outcomes in women with transposition of the great arteries and arterial switch operation. Am J Cardiol 2010: 106; 417-420.

[8] Hinze A, Kutty S, Sayles H, Sandene EK, Meza J , Kugler JD. Reproductive and contraceptive counseling received by adult women with congenital heart disease: A Risk-based analysis. Congenital Heart Disease 2013: 8; 20-31.

[9] Vigl M, Kaemmerer $M$, Seifert-Klauss $V$, Niggemeyer E, Nagdyman $N$, Trigas $V$, Bauer $U$, Schneider K-TM , Berger F , Hess J , Kaemmerer H. Contraception in women with congenital heart disease. Am J Cardiol 2010: 106; 1317-1321.

[10] Thorne S, MacGregor A, Nelson-Piercy C. Risks of contraception and pregnancy in heart disease. Heart 2006: 92; 1520-1525.

[11] Silversides C, Sermer M, Siu S. Choosing the best contraceptive method for the adult with congenital heart disease. Curr Cardiol Rep 2009: 11; 298-305.

[12] Center for Disease Control and Prevention, U.S. Medical Eligibility Criteria for Contraceptive Use 2010. Adapted from the WHO Medical eligibility criteria for contraceptive use (2010). 
[13] American College of Obstetricians and Gynecologists, Practice Bulletin No. 121: LongActing Reversible Contraception: Implants and Intrauterine Devices, Obstetrics \& Gynecology. 118 (2011) 184-196.

[14] Daniels K, Daugherty J, Jones J. Current Contraceptive Status Among Women Aged 15-44: United States, 2011-2013. NCHS data brief 2014: 173.

[15] Eeckhaut MCW. Marital status and female and male contraceptive sterilization in the United States. Fertility and Sterility 2015: 103; 1509-1515.

[16] Mosher WD , Jones J Abma JC. Intended and Unintended Births in the United States: 1982-2010. National Health Statistics Reports 2012: 55.

[17] US Department of Health \& Human Services. CDC Vital \& Health Statistics: Use of contraception in the U.S.: 1982-2008. 2010.

[18] Petitti DB. Hormonal contraceptives and arterial thrombosis - Not risk-free but safe enough. NEngl J Med 2012: 336; 2316-2318.

[19] Pijuan-Domènech $A$, Baró-Mariné $F$, Rojas-Torrijos $M$, Dos-Subirà L , Pedrosa-Del Moral V, Subirana-Domènech MT , Goya-Canino M , Cabero-Roura L, Garcia-Dorado D, CasaldàligaFerrer $\mathrm{J}$. Usefulness of progesterone-only components for contraception in patients $w$ ith congenital heart disease. Am J Cardiol 2013: 112; 590-593 
[20] Potter BJ, Leong-Sit P, Fernandes SM, Feifer A, Mayer JE , Triedman JK, Walsh EP, Landzberg MJ, Khairy P. Effect of aspirin and warfarin therapy on thromboembolic events in patients with univentricular hearts and Fontan palliation. Int J Cardiol 2013: 168; 3940-3943.

[21] Haeffele C, Lui G. Dextro-transposition of the great arteries: Long-term sequelae of atrial and arterial switch. Cardiol Clin 2015: 33; 543-558. 
Highlights:

- Unplanned pregnancies in women with congenital heart disease

- Adverse events from oral contraception

- Contraception counseling

- Complex congenital heart disease thrombotic risk substrates

Keywords: congenital heart disease, contraception, pregnancy, Fontan, thrombosis

Acknowledgements: This was a multi-center project of the Adult Congenital Cardiac Care Associate (ACCCA) Research Group. We gratefully acknowledge the assistance of Rachel Bolanos, MPH; Kirstie MacMillen, BS; Linda Houser, RN, MS, NP; Miranda Zinsman, B.S.; Haley Hedlin, Ph.D 